American Medical Journal 3 (2): 175-182, 2012

ISSN 1949-0070

(C) 2012 Science Publications

\title{
Dietary Fats and Heart Disease: From Prenatal to Postnatal Environment
}

\author{
Sukhinder K. Cheema \\ Department of Biochemistry, \\ Memorial University, St. John's, NL, Canada A1B 3X9, Canada
}

\begin{abstract}
Problem statement: Heart disease is the leading cause of death worldwide and is dramatically increasing in developing countries. Preventive approaches, such as dietary and lifestyle changes can have major health implications to prevent the onset of heart disease. Besides healthy nutrition in the adult life, maternal nutrition during pregnancy and lactation can also have a significant impact on the outcome of heart disease in the later life of the offspring. Approach: Higher levels of plasma total cholesterol, especially Low-Density Lipoprotein (LDL) cholesterol and lower levels of high-density lipoprotein cholesterol are associated with an increased risk of heart disease. We have used various animal models and dietary strategies to investigate the regulation of lipid and lipoprotein metabolism. We also manipulated maternal diets during pregnancy and lactation to study the outcome of heart disease in the offspring in later life. Results: Reducing the intake of saturated and trans fatty acids reduce the risk of heart disease, while polyunsaturated fatty acids elicit beneficial health effects. Amongst polyunsaturated fatty acids, omega-3 class of fatty acids, are associated with lower incidence of heart disease however, an increased consumption of omega- 6 fatty acids may increase the risk of heart disease. Higher intake of saturated fat during pregnancy and lactation inhibits the gene expression of LDL receptor which leads to an increase in plasma LDL-cholesterol levels of the offspring thereby putting the offspring at a higher risk of heart disease. Conclusion/Recommendations: An optimum ratio of omega- 6 to omega-3 fatty acids is critical to reduce the risk of heart disease. Developing countries need to pay a special attention to dietary guidelines to reduce the burden of heart disease.
\end{abstract}

Key words: Dietary fats, prenatal and postnatal nutrition, cardiovascular disease, lipids and cholesterol

\section{INTRODUCTION}

Heart disease is the leading cause of death in not only the developed countries but also in developing countries such as India. A recent report suggests that by the year 2010, India will bear $60 \%$ of the world's heart disease burden (Khunti and Samani, 2004; Anand et al., 2000; Kahn, 2008) having more than 100 million people affected by the disease. It was also reported that the average age of patients in India, with heart disease, is significantly lower when compared to people in other developed countries. In the past five decades, the rates of heart disease among urban populations in India have risen from 4-11\% (Khunti and Samani, 2004; Anand et al., 2000). Thus heart disease, which was once considered as the disease of the Western countries, is now over powering India. The question arises as to why India's health worries have now shifted from age-old problems, such as infectious disease, famine and malnutrition, towards heart disease, obesity and metabolic syndrome? One of the reasons may be the road to modernization, where a larger number of populations are trading healthy traditional diets for fattier foods and a sedentary lifestyle. Besides poor dietary habits, the Indian population also appears to have a genetic makeup that predisposes them to heart disease (Anand et al., 2000). South Asians have elevated levels of Low-Density Lipoprotein (LDL)cholesterol ("bad cholesterol") and triglycerides that are associated with an increased risk of heart disease, while the concentrations of High-Density Lipoprotein (HDL)cholesterol ("good cholesterol"), which is protective against heart disease, are lower. Moreover, the South Asian population tends to gain weight in the abdominal region which increases the risk of heart disease (Anand et al., 2000). Lastly, the nutrition of the mothers, during pregnancy and lactation, may also contribute towards early onset of diseases, such as heart disease, diabetes and obesity, in the offspring (Chechi et al., 2009; McMillen and Robinson, 2005; McMillen et al., 2008). Animal studies have confirmed that feeding high fat diets during gestation and lactation leads to abnormalities of metabolic pathways of the offspring thereby predisposing the offspring to higher incidence of diseases in later life. These reports stress the importance of nutrition awareness in the general population in India 
for the prevention and treatment of heart disease and related diseases. A focus on prevention will certainly reduce the health care cost in the future.

Dietary fats and heart disease: Dietary fats are essential for the growth and development, however an excess of fat can cause the onset of heart disease and related complications (Watts et al., 1996). Dietary fat intake has long been associated with an increased risk of heart disease, where both the quality and the quantity of fat are known to play an important role. The influence of fat on the state of the body and mind was depicted as early as the 18th century by a Swiss physiologist, Albrecht von Haller, who had stated that "During sleep and when the body and spirit are in response, fat deposits in the cells and when it is in too great quantity it becomes injurious because it compresses the blood vessels and resists the action of heart. This makes one subject to asthma, stroke and dropsy" (Carpenter, 1998). The importance of dietary fats in cardiovascular disease first emerged in 1953 when Ancel Keys conducted the first worldwide epidemiological study to link dietary fats to mortality from heart disease (Keys, 1953). In the "seven countries" study, Keys (Keys, 1970) found significant association between total fat and saturated fat intake and mortality from heart disease. The association between dietary fats and heart disease is mainly due to alterations in blood triglyceride and cholesterol levels. The first guidelines on consumption of dietary fat were published in (Page et al., 1957) and amended in (Page et al., 1961) that generally reflected the guidelines recognized today. In 1968, the Committee on Nutrition of the American Heart Association published guidelines to reduce the intake of animal fat, decrease saturated fats and increase polyunsaturated fats and to apply dietary recommendations early in life.

The major kinds of fats consumed in our diet are saturated, polyunsaturated, monounsaturated and trans fatty acids. The most abundant fatty acids found in nature have an even number of carbon atoms and the chain length varies from four to 30 carbons long. The most abundant fatty acids found in food are 18-carbons long. The nomenclature of fatty acids is based on the number of carbon atoms in the chain, the degree of unsaturation and the location of the double bond. The position of the first double bond nearest from the methyl end of the carbon chain is described by an omega number also referred to as $n$ or $\omega(n-3, n-6, n-7$ and n-9). Polyunsaturated fatty acids with the first double bond three carbons away from the methyl end is an n-3 fatty acids, whereas polyunsaturated fatty acids with its first double bond six carbons away from the methyl end is an n-6 fatty acid. Humans are not able to synthesize n-6 (linoleic acid; 18:2) or n-3 (linolenic acid; 18:3) fatty acids, making these fatty acids essential dietary nutrients that must be consumed in our diet. Table 1 gives the most common fatty acids found in nature, the number and position of the double bond and the common foods containing these fatty acids.

Saturated and trans fats in Heart Disease: The consumption of both saturated and trans fatty acids have a positive correlation with the onset of heart disease (Watts et al., 1996; Denke, 2006). Studies have shown that these fatty acids increase the levels of blood total- and LDL-cholesterol (Spady et al., 1993). The plasma LDL-cholesterol levels increase by $1.3-1.7 \mathrm{mg}$ $\mathrm{dL}^{-1}$ for every $1 \%$ increase in energy from saturated fatty acids (Keys et al., 1965; Mensink and Katan, 1992). Studies have also shown a dose-dependent effect of trans fatty acids in increasing plasma LDLcholesterol concentrations (IM, 2005). Saturated fats are usually solid at room temperature and are not highly prone to oxidation when compared with unsaturated fats. Trans fats, on the other hand, are unsaturated, but they can raise total and LDL-cholesterol and lower HDL-cholesterol concentrations, similar to the effects of saturated fatty acids, thereby increasing the risk of heart disease (Mensink and Katan, 1992). Although naturally occurring in some foods such as dairy and animal meats, trans fats generally result from baking, cooking and frying in most restaurants and fast-food chains. Meat and full-fat dairy products contribute approximately $60 \%$ of saturated fatty acids in a typical diet. Other main sources of saturated fat and trans fat in a typical Indian diet are foods from some plants and the consumption of deep fried foods. In most restaurants, the cooking oil after frying is not changed frequently enough to reduce the intake of oxidized fat, which is also linked to an increased risk of heart disease (Ghafoorunissa, 2008). In certain parts of India, trans fats from hydrogenated vegetable oil, commonly known as vanaspati, are consumed to much greater quantity than in the United States thereby increasing the risk of heart disease.

Table 1: Common fatty acids found in nature

\begin{tabular}{llllll}
\hline Fatty acid & Notation & $\begin{array}{l}\text { Number of } \\
\text { double bounds }\end{array}$ & $\begin{array}{l}\text { Position of the } \\
\text { double bound }\end{array}$ & Saturation & $\begin{array}{l}\text { Common food } \\
\text { sources }\end{array}$ \\
\hline Stearic acid & $18: 00$ & 0 & - & Saturated & Most animal fats \\
Oleic acid & $18: 01$ & 1 & n-9 & Pounsaturated & Olive oil.canola oil \\
Linoleic acid & $18: 02$ & 2 & n-6 & Polyunsaturated & Sunflower.Safflower.corn oils \\
Linolenic acid & $18: 03$ & 3 & Soybean oils.flax oil & \\
\hline
\end{tabular}


The North American dietary guidelines have regulations in place for the consumption of dietary fats and the type of fat in the diet. It is recommended to limit the intake of foods high in saturated fat, trans fat and cholesterol, such as whole-milk dairy products, fatty meats, partially hydrogenated vegetable oils, fried foods and egg yolks. Instead, it is recommended to choose foods low in saturated fat, trans fat and cholesterol. This can be accomplished by enriching the diet in fruits and vegetables, grain products especially from whole grains, lentils and beans and to add either fish or flax powder (ground alsi) to the diet. The intake of saturated fat should not exceed $7 \%$ of total calories each day; trans fat intake should not exceed $1 \%$ of total calories each day. The intake of total fat (saturated, trans, monounsaturated, polyunsaturated) should be no more than $30 \%$ of total calories, while that of saturated + trans fat should be no more than $10 \%$ of total calories.

Unsaturated fats and the risk of heart disease: Both mono- and polyunsaturated fatty acids are considered healthy fats as these are known to lower the risk of heart disease. Oleic acid, a monounsaturated fatty acid, lowers total and LDL-cholesterol concentrations when it replaces saturated fatty acids (IM, 2005; KrisEtherton, 1999). Similarly, studies have shown that an increase of $1 \%$ energy from polyunsaturated fatty acids reduces the levels of total cholesterol by $0.9 \mathrm{mg} \mathrm{dL}^{-1}$ (Keys et al., 1965; Hegsted et al., 1965). Clinical studies have shown that polyunsaturated fatty acids decreased the risk of heart disease by $25-43 \%$ (Dayton et al., 1969; Leren, 1970; Singh et al., 2002). Unsaturated fats have at least one unsaturated bond or a double bond and are often found in liquid oils of vegetable origin. Polyunsaturated fats are easily oxidized and become rancid, especially under high temperatures such as heating. Monounsaturated fats are also liquid at room temperature, however can start to solidify when kept in the refrigerator. Although both types of unsaturated fats may help lower blood cholesterol level when used in place of saturated fats in the diet, the total amount of fat should still not exceed $30 \%$ of the calories of the total daily intake. Some of the rich sources of oils rich in polyunsaturated fatty acids are: safflower oil, mustard oil, corn oil, sesame oil, sunflower oil and nuts/seeds. On the other hand, the oils rich in monounsaturated fats are: olive oil, peanut oil, avocado oil.

Omega-3 polyunsaturated fatty acids in heart disease: Long chain polyunsaturated fatty acids of the omega-3 (n-3) series, such as Eicosapentaenoic Acid
(EPA) and Docosahexaenoic Acid (DHA), are found to a great extent in fish, fish oil and other marine sources. These n-3 fatty acids are known to have cardioprotective effects due to their antiarrythmic, antiinflammatory and antiatherogenic properties (Demaison and Moreau, 2002). The relationship between fish and fish oil consumption and reduced risk of heart disease was first observed by Bang et al. (1971) who found that Eskimos were at a lower risk of coronary heart disease compared to Danes. The beneficial effects of fish oil are mainly attributed to the biological effects of n-3 PUFA, such as EPA and DHA (Dyerberg, 1986; Kagawa et al., 1982). Consumption of two meals/week of a fatty fish over two years reduced coronary artery infarction by $29 \%$ in human subjects (Burr et al., 1989). One of the biggest challenges is however to decide the appropriate ratio of n-6 to n-3 fatty acids. It is generally accepted that the optimum ratio should be between 4-10:1. If the diet contains a ratio of $n-6 / n-3$ fatty acids greater than $50: 1$, one can expect to develop n-3 deficiency along with an increased risk of heart disease and other complications; this appears to be the current ratio consumed by the general public.

Awareness in the general population in North America to increase the intake of polyunsaturated fatty acids and to decrease the intake of saturated fat caused a dramatic increase in the consumption of vegetable oils, which are significantly rich in $n-6$ fatty acids. The consumption of $n-3$ rich sources however remained the same or decreased, which resulted in a significant increased ratio of n-6 to n-3 fatty acids (Simopoulos, 2006). The only exception to this trend is perhaps in the Japanese and Eskimos (Dyerberg, 1989) population, where the intake of n-3 fatty acids is very high and can reach up to $10 \mathrm{~g} \mathrm{day}^{-1}$. Decreased intake of $\mathrm{n}-3$ fatty acids is not only linked to the increased incidence of heart disease but it also increases the risk of asthma, arthritis, other inflammatory diseases and certain forms of cancer. Compared to the present very low intake of n-3 polyunsaturated fatty acids in most countries, it would require a large increase in the intake of fatty fish or alternative sources of EPA and DHA. Approximately 100-200 g of fatty fish (herring, mackerel, fresh tuna, sardines and cod, salmon) provides about $2 \mathrm{~g}$ of very long chain n-3 polyunsaturated fatty acids. On the other hand, consuming only $1 \mathrm{~g}$ of fish oil capsules provides about 200-300 mg of EPA and DHA. Caution should however be taken when using fish oil supplements as high intakes of n-3 fatty acids can lead to side effects, especially increased bleeding. There is also some evidence that high intakes of n-3 PUFA increase the requirements for vitamin $\mathrm{E}$, possibly because of potential increase in lipid peroxidation. Besides fish and 
fish oil, flaxseed (alsi) contains high amounts of linolenic acid, an n-3 fatty acid that is converted to EPA and DHA in human body, thus making it a perfect alternative of n-3 fatty acids for vegetarians. Other vegetarian sources of $n-3$ polyunsaturated fatty acids are canola oil and walnuts.

Nutrition during pregnancy and the risk of heart disease in the offspring in adult life: Metabolic problems associated with the consumption of dietary fats are not only important for the health of an individual but also for the health of the offspring born to mothers consuming specific nutrients during pregnancy and lactation. The 'fetal origins hypothesis' known more appropriately as the Developmental Origins of Health and Disease (DOHaD) hypothesis suggests that nutrition during early or prenatal life has permanent effects on the cardiovascular health of a person in adult life (Barker, 1995; 1997). It is proposed that adverse nutrition in the womb creates a 'stressed environment' for the developing fetus. The fetus responds to nutritional stress by 'programming' its own growth in a way that puts the offspring at an increased risk of future metabolic disorders (Fig. 1). Studies have employed maternal nutrient restriction models to induce the metabolic abnormalities like insulin resistance (Snoeck et al., 1990), dyslipidemia (Khan et al., 2005) and hypertension (Sherman and Langley-Evans, 2000) in the offspring. However, there is limited data from epidemiological and animal studies concerning overnutrition, especially the consumption of excess calories from fat, during pregnancy or early life and its effects on health in later life.

The incidences of metabolic syndrome and cardiovascular disease are steadily increasing in the traditional Western societies where maternal undernutrition is not a primary concern. A typical Western diet is rich in dietary fat content, especially saturated fatty acids, which is linked to an increased incidence of heart disease (Keys et al., 1965). There are only a few animal studies to date indicating that high fat consumption during pregnancy can induce features of metabolic syndrome in the adult offspring independent of the adult environmental factors (Taylor et al., 2005; Guo and Jen, 1995). A recent study from our research group has shown that maternal diet rich in saturated fatty acids caused significant inhibition of hepatic LDLreceptor gene in the offspring of mice, which was responsible for an increase in plasma total- and LDLcholesterol concentrations (Anand et al., 2000). It was also observed that the maternal diets rich in saturated fatty acids caused endothelial dysfunction of the aortae in the offspring. Other studies have shown that trans fatty acids get deposited in infant tissues, thereby causing alterations in the metabolic pathways to put the infant at higher risk of disease (Dalainas and Ioannou, 2008). Combined together, these findings suggest that maternal intake of saturated and trans fatty acids may put the offspring at an increased risk of heart disease in later life.

The fatty acid composition of maternal diets can alter the regulation of several transcription factors involved in the regulation of metabolic pathways (Jump, 2008). It is suggested that epigenetic regulation of gene transcription involving DNA methylation and histone modifications are key factors in regulating early development (Heerwagen et al., 2010). Peroxisome Proliferatoractivated Receptors (PPARs) are a class of transcription factors that regulate lipid, cholesterol and glucose metabolism and are known to be under the control of epigenetic regulation (Lillycrop et al., 2008; McCurdy et al., 2009). Thus dietary fats during pregnancy can have long-term effects that involve DNA modification.

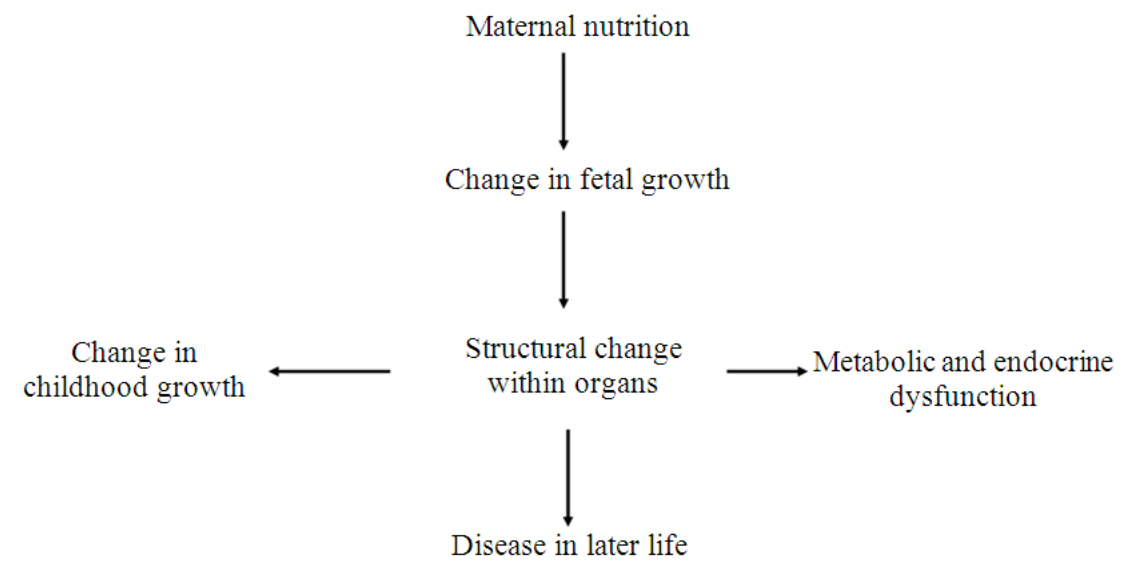

Fig. 1: Development origins of health and disease and the importance of maternal nutrition 
Omega-3 fatty acids during pregnancy: As opposed to saturated fats, polyunsaturated fats, especially those from the omega-3 group, have been shown to induce beneficial effects. The consumption of polyunsaturated fatty acids during pregnancy and lactation influence the transfer of these fatty acids across the placenta and through breast milk thereby providing health benefits (Innis et al., 2006; Innis, 2004). A higher intake of omega-3 fatty acids by the mothers increases the transfer of these fatty acids to the infant before birth, causing protective effects. Several reports have established long-term benefits of omega-3 fatty acids, especially DHA, on the child's development. The levels of blood DHA in infants has been shown to be associated with higher scores on measures of neural and visual maturation (Ceruku et al., 2002; Jorgensen et al., 2001). Moreover, children born to mothers who consumed seafood during pregnancy showed higher verbal intelligence (Hibbeln et al., 2007). The consumption of seafood or fish is low amongst North Indian population however this population can gain significant health benefits from consuming flax, which is a good source of omega-3 fatty acid Alpha-Linoleic Acid (ALA). The question arises whether dietary ALA can provide sufficient amounts of higher chain omega-3 fatty acids through the elongation and desaturation pathways. It appears that the conversion of ALA to DHA is low in humans (Pawlosky et al., 2003; Hussein et al., 2005). On the other hand, newborn infants can convert significant amounts of ALA to EPA and DHA (Salem et al., 1996). Moreover, the conversion of ALA to DHA is higher in women than men, which is further increased during pregnancy (Williams and Burdge, 2006). Incorporating flax in the diet will likely induce beneficial health effects thus it should be encouraged as a health food.

Genetic makeup and heart disease: South Asians seem to be naturally predisposed to heart disease compared to other ethnic groups (Anand et al., 2000). South Asians, living in Canada, have a higher risk of heart disease when compared to the European and Chinese population, even after adjusting for all other known risk factors. This is suggested to be due to a "thrifty-gene" in South Asians who adapted over many generations to the region's frequent famines. However an overabundance of food in the present days is causing imbalances of metabolic pathways leading to an increased risk of heart disease, insulin resistance, diabetes and obesity. The levels of LDL-cholesterol in South Asians are similar to other populations however they tend to carry small-dense LDL, which is considered to be more atherogenic (Kulkarni et al.,
1999) due to its increased susceptibility to oxidation. On the other hand, the South Asian population not only has lower levels of HDL-cholesterol, the "good cholesterol", but they also have a higher concentration of small, less protective HDL particles (Bhalodkar et al., 2004).

Besides having a higher risk for heart disease, the prevalence of diabetes mellitus and obesity, other risk factors for the onset of heart disease, is uniformly higher in South Asians. It is projected that India alone will experience the greatest global increase in type 2 diabetes by the year 2025 (King et al., 1998). The prevalence of diabetes is low in the rural areas, where only about $2 \%$ of the population is diabetic however the risk is much higher in urban population. The risk of type 2 diabetes is even greater amongst the Indian population settled in the Western World (Venkataraman et al., 2004). The Indian population also appears to have greater fat deposits in the abdominal area, which again puts them at a higher risk of heart disease. Recent studies suggest that the waist/hip ratio may provide a better estimate of the degree of abdominal obesity and the risk of heart disease (Yusuf et al., 2005). Visceral or abdominal obesity is also an important factor in the pathogenesis of insulin resistance and heart disease.

\section{CONCLUSION}

Several studies have strongly indicated that the Indian population is predisposed to a higher risk of heart disease and the related risk factors. It is thus important for the Indian population to pay a greater attention to their lifestyle and diet. It is further noted that certain diets may induce greater detrimental health effects in a population that is already predisposed to a disease. The association between genetic makeup of an individual, the response to a particular diet and the onset of disease is an emerging area of research. This is covered under both nutrigenetics (response of an individual to a particular nutrient based on one's genetic makeup) and nutrigenomics (alterations in gene expression by various nutrients) to design personalized diets for better health (Fig. 2). It is likely that within the next few years, experts on nutrigenomics will be able to precisely tailor a diet for one's specific needs. This may help in preventing or delaying the onset of heart disease and related diseases. It is important to control the type and amount of different fats in one's diet. In general, eating a healthy diet that is low in fat (less than 30\% of total caloric intake from fat), especially in saturated and trans fats (less than $10 \%$ of total caloric intake from fat), along with consumption of plenty of vegetables and fruits, is recommended for a healthy lifestyle. 


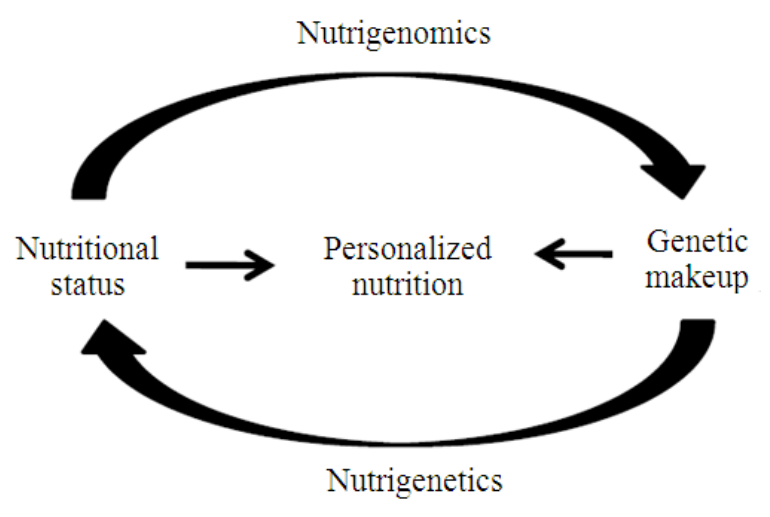

Fig. 2: Nutrigenomics focuses on the effect of nutrients on the genome, whereas nutrigenetics focuses on the effect of genetic variation on the response to a particular nutrient

For individuals who do not consume fish, other options are available such as consuming flax, walnuts and canola oil, to consume "designer" foods high in omega3 fatty acids or to take omega-3 supplements. The omega-6 to omega-3 fatty acid ratio should be maintained in the range of 5-10:1 for beneficial health effects. Finally, attaining proper health is in one's own hands by following good eating habits and exercising regularly.

\section{ACKNOWLEDGEMENT}

Research is supported by funds from Natural Sciences and Engineering Research Council (NSERC), Canadian Institutes of Health Research (CIHR) and Heart \& Stroke Foundation of Canada (HSFC).

\section{REFERENCES}

Anand, S.S., S. Yusuf, V. Vuksan, S. Devanesen and K.K. Teo et al., 2000. Differences in risk factors, atherosclerosis and cardiovascular disease between ethnic groups in Canada: The Study of Health Assessment and Risk in Ethnic groups (SHARE). Lancet, 356: 279-284. PMID: 11071182

Bang, H.O., J. Dyerberg and A.B. Nielsen, 1971. Plasma lipid and lipoprotein pattern in Greenland West-Coast Eskimos. Lancet, 1: 1143-1145. DOI: 10.1016/S0140-6736(71)91658-8

Barker, D.J., 1995. Fetal origins of coronary heart disease. Br. Med. J., 311: 171-174. PMID: 7613432

Barker, D.J.P., 1997. Fetal nutrition and cardiovascular disease in later life. Br. Med. Bull., 53: 96-108.
Bhalodkar, N.C., S. Blum, T. Rana, A. Bhalodkar and R. Kitchappa et al., 2004. Comparison of levels of large and small high density lipoprotein cholesterol in Asian Indian men compared with Caucasian men in the Framingham Offspring Study. Am. J. Cardiol., 94: 1561-1563. DOI: 10.1016/j.amjcard.2004.08.040

Burr, M.L., A.M. Fehily, J.F. Gilbert, S. Rogers and R.M. Holliday et al., 1989. Effects of changes in fat, fish and fibre intakes on death and myocardial reinfarction: Diet and Reinfarction Trial (DART). Lancet, 2: 757-761. PMID: 2571009

Carpenter, K.J., 1998. Early ideas on the nutritional significance of lipids. J. Nutr., 128: 423S-426S. PMID: 9478041

Ceruku, S.R., H.E. Montegomery-Downs, S.L. Farkas, E.B. Thomas and C.J. Lammi-Keefe, 2002. Higher maternal plasma docosahexaenoic acid during pregnancy is associated with more mature neonatal sleep-state patterning. Am. J. Clin. Nutr., 76: 608613.

Chechi, K., J.J. McGuire and S.K. Cheema, 2009. Developmental programming of lipid metabolism and aortic vascular function in C57BL/6 mice: $\mathrm{A}$ novel study suggesting an involvement of LDLreceptor. Am. J. Physiol. Regul. Integr. Comp. Physiol., 296: R1029-R1040. PMID: 19193942

Dalainas, I. and H.P. Ioannou, 2008. The role of trans fatty acids in atherosclerosis, cardiovascular disease and infant development. Int. Angiol., 27: 146-156. PMID: 18427401

Dayton, S., M.L. Pearce, S. Hashimoto, W.J. Dixon and U. Tomiyasu, 1969. A controlled clinical trial of a diet high in unsaturated fat in preventing complications of atherosclerosis. Circulation, 40: $1-63$.

Demaison, L. and D. Moreau, 2002. Dietary n-3 polyunsaturated fatty acids and coronary heart disease-related mortality: a possible mechanism of action. Cell Mol. Life Sci., 59: 463-77. DOI: 10.1007/s00018-002-8439-1

Denke, M.A, 2006. Dietary fats, fatty acids and their effects on lipoproteins. Curr. Atheroscler. Rep., 8: 466-471. DOI: $10.1007 / \mathrm{s} 11883-006-0021-0$

Dyerberg, J., 1986. Linolenate-derived polyunsaturated fatty acids and prevention of atherosclerosis. Nut. Rev., 44: 125-134. DOI: 10.1111/j.17534887.1986.tb07603.x

Dyerberg, J., 1989. Coronary heart disease in Greenland Inuit: a paradox. Implications for western diet patterns. Arctic. Med. Res., 48: 47-54. PMID: 2736000 
Ghafoorunissa, G., 2008. Role of trans fatty acids in health and challenges to their reduction in Indian foods. Asia Pac. J. Clin. Nut., 1: 212-215. PMID: 18296340

Guo, F. and K.L. Jen, 1995. High-fat feeding during pregnancy and lactation affects offspring metabolism in rats. Physiol. Behav., 57: 681-686. PMID: 7777603

Heerwagen, M.J., M.R. Miller, L.A. Barbour and J.E. Friedman, 2010. Maternal obesity and fetal metabolic programming: A fertile epigenetic soil. Am. J. Physiol. Regul. Integ. Comp. Physiol., 299: R711-R722. PMID: 20631295

Hegsted, D.M., R.B. McGandy, M.L. Myers and E.J. Stare, 1965. Quantitative effects of dietary fat on serum cholesterol in man. Am. J. Clin. Nutr., 17: 281-295.

Hibbeln, J.R., J.M. Davis, C. Steer, P. Emmett and I. Rogers et al., 2007. Maternal seafood consumption in pregnancy and neurodevelopmental outcomes in childhood (ALSPAC study): An observational cohort study. Lancet, 369: 578-585. PMID: 17307104

Hussein, N., E. Ah-Sing, P. Wilkinson, C. Leach and B.A. Griffin et al., 2005. Long-chain conversion of [13C]linoleic acid and alpha-linolenic acid in response to marked changes in their dietary intake in men. J. Lipid Res., 46: 269-280. PMID: 15576848

IM, 2005. Dietary Reference Intakes for Energy, Carbohydrate, Fiber, Fat, Fatty Acids, Cholesterol, Protein and Amino Acids. 1st Edn., National Academies Press, Washington DC, ISBN-10: 030908525X, pp: 1045.

Innis, S.M., 2004. Polyunsaturated fatty acids in human milk: An essential role in infant development. Adv. Exp. Med. Biol., 554: 27-43. PMID: 15384565

Innis, S.M., J. Palaty, Z. Vaghri and G. Lockitch, 2006. Increased levels of mercury associated with high fish intakes among children from Vancouver, Canada. J. Pediatrics, 148: 759-763. PMID: 16769382

Jorgensen, M.H., O. Hernell, E. Hughes and K.F. Michaelsen, 2001. Is there a relation between docosahexaenoic acid concentration in mothers' milk and visual development in term infants? J. Pediatric. Gastroenterl. Nutr., 32: 293-296. PMID: 11345178

Jump, D.B., 2008. N-3 polyunsaturated fatty acid regulation of hepatic gene transcription. Curr. Opin. Lipidology., 19: 242-247. PMID: 18460914
Kagawa, Y., M. Nishizawa, M. Zuzuki, T. Miyatake and T. Hamamoto et al., 1982. Eicosapolyenoic acids of serum lipids of Japanese islanders with low incidence of cardiovascular diseases. J. Nut. Sci. Vitaminol (Tokyo), 28: 441- 453. PMID: 7175583

Kahn, D., 2008. Getting to the heart of the matter in India. Lancet, 372: 523-524. DOI: 10.1016/S01406736(08)61217-9

Keys, A., 1953. Atherosclerosis: A problem in newer public health. J. Mt. Sinai. Hosp N. Y., 20: 118139. PMID: 13085148

Keys, A., 1970. A practical, palatable and prudent way of eating. J. Med. Assoc. Ga., 59: 355-359. PMID: 5452772

Keys, A., J.T. Anderson and F. Grande, 1965. Serum cholesterol response to changes in the diet: IV. Particular saturated fatty acids in the diet. Metabolism, 14: 776-787. DOI: 10.1016/00260495(65)90004-1

Khan, I.Y., V. Dekou, G. Douglas, R. Jensen and M.A. Hanson et al., 2005. A high-fat diet during rat pregnancy or suckling induces cardiovascular dysfunction in adult offspring. Am. J. Physiol. Regul. Integr. Comp. Physiol., 288: R127-R133. PMID: 15308487

Khunti, K. and N.J. Samani, 2004. Coronary heart disease in people of south-Asian origin. Lancet, 364: 2077-2078. DOI: $10.1016 /$ S01406736(04)17563-6

King, H., R.E. Aubert and W.H. Herman, 1998. Global burden of diabetes, 1995-2025: Prevalence, numerical estimates and projections. Diabetes Care, 21: 1414-1431. PMID: 9727886

Kris-Etherton, P.M., 1999. AHA Science Advisory: Monounsaturated fatty acids and risk of cardiovascular disease. Circulation, 100: 12531258.

Kulkarni, K.R., J.H. Markovitz, N.C. Nanda and J.P. Segrest, 1999. Increased prevalence of smaller and denser LDL particles in Asian Indians. Arterioscler. Thromb. Vasc. Biol., 19: 2749-2755. PMID: 10559021

Leren, P., 1970. The Oslo diet-heart study: Eleven-year report. Circulation, 42: 935-942. DOI: 10.1161/ 01.CIR.42.5.935

Lillycrop, K.A., E.S. Phillips, C. Torrens, M.A. Hanson and A.A. Jackson et al., 2008. Feeding pregnant rats a protein-restricted diet persistently alters the methylation of specific cytosines in the hepatic PPAR alpha promoter of the offspring. Br. J. Nut., 100: 278-282. PMID: 18186951 
McCurdy, C.E., J.M. Bishop, S.M. Williams, B.E. Grayson and M.S. Smith et al., 2009. Maternal high-fat diet triggers lipotoxicity in the fetal livers of nonhuman primates. J. Clin. Invest., 119: 323335. PMID: 19147984

McMillen, I.C. and J.S. Robinson, 2005. Developmental origins of the metabolic syndrome: Prediction, plasticity and programming. Physiol. Rev., 85: 571-633.

McMillen, I.C., S.M. MacLaughlin, B.S. Muhlhausler, S. Gentili and J.L. Duffield et al., 2008. Developmental origins of adult health and disease: The role of periconceptional and foetal nutrition. Basic Clin. Pharmacol. Toxicol., 102: 82-89. PMID: 18226059

Mensink, R.P. and M.B. Katan, 1992. Effect of dietary fatty acids on serum lipids and lipoproteins. A meta-analysis of 27 trials. Arterioscler. Thromb., 12: 911-919.

Page, I.H., E.V. Allen, F.L. Chamberlain, A. Keys and J. Stamler et al., 1961. Dietary fat and its relation to heart attacks and strokes. Circulation, 23: 133136.

Page, I.H., F.J. Stare, A.C. Corcoran, H. Pollak and C.F. Wilkinson Jr., 1957. Atherosclerosis and the fat content of the diet. Circulation, 16: 164-178.

Pawlosky, R.J., J.R. Hibbeln, Y. Lin, S. Goodson and P. Riggs et al., 2003. Effects of beef- and fishbased diets on the kinetics of n-3 fatty acid metabolism in human subjects. Am. J. Clin. Nutr., 77: 565-572. PMID: 12600844

Salem, N. Jr., B. Wegher, P. Mena and R. Uauy, 1996. Arachidonic and docosahexaenoic acids are biosynthesized from their 18-carbon precursors in human infants. Proc. Natl. Acad. Sci. USA., 93: 49-54. PMID: 8552667

Sherman, R.C. and S.C. Langley-Evans, 2000. Antihypertensive treatment in early postnatal life modulates prenatal dietary influences upon blood pressure in the rat. Clin. Sci. (Lond), 98: 269-75. PMID: 10677384

Simopoulos, A.P., 2006. Evolutionary aspects of diet, the omega-6/omega-3 ratio and genetic variation: Nutritional implications for chronic diseases. Biomed. Pharmacother., 60: 502-507. PMID: 17045449
Singh, R.B., G. Dubnov, M.A. Niaz, S. Ghosh and R. Singh et al., 2002. Effect of an Indo-Mediterranean diet on progression of coronary disease in high risk patients: a randomized single blind trial. Lancet, 360: 1455-1461. DOI: 10.1016/S01406736(02)11472-3

Snoeck, A., C. Remacle, B. Reusens and J.J. Hoet, 1990. Effect of a low protein diet during pregnancy on the fetal rat endocrine pancreas. Biol. Neonate, 57: 107-118. PMID: 2178691

Spady, D.K., L.A. Woollett and J.M. Dietschy, 1993. Regulation of plasma LDL-cholesterol levels by dietary cholesterol and fatty acids. Annu. Rev. Nutr., 13: 355-381. DOI: 10.1146/annurev.nu.13.070193.002035

Taylor, P.D., J. McConnell, I.Y. Khan, K. Holemans and K.M. Lawrence et al., 2005. Impaired glucose homeostasis and mitochondrial abnormalities in offspring of rats fed a fat-rich diet in pregnancy. Am. J. Physiol. Regul. Integr. Comput. Physiol., 288: R134-R139. DOI: 10.1152/ajpregu.00355. 2004

Venkataraman, R., N.C. Nanda, G. Baweja, N. Parikh and V. Bhatia, 2004. Prevalence of diabetes mellitus and related conditions in Asian Indians living in the United States. Am. J. Cardiol., 94: 977-980. PMID: 15464696

Watts, G.F., P. Jackson, V. Burke and B. Lewis, 1996. Dietary fatty acids and progression of coronary artery disease in men. Am. J. Clin. Nutr., 64: 202209. PMID: 8694021

Williams, C.M. and G.C. Burdge, 2006. Long-chain n-3 PUFA: Plant v. marine sources. Proc. Nutr. Soc., 65: 42-50. PMID: 16441943

Yusuf, S., S. Hawken, S. Ounpuu, L. Bautista and M.G. Franzosi et al., 2005. Obesity and the risk of myocardial infarction in 27,000 participants from 52 countries: A case-control study. Lancet, 366: 1640-1649. PMID: 16271645 